\title{
The effectiveness of different sweeteners in suppressing citric acid sourness
}

\author{
HENDRIK N. J. SCHIFFERSTEIN and JAN E. R. FRIJTERS \\ Agricultural University, Wageningen, The Netherlands
}

\begin{abstract}
The exact mechanism that causes taste suppression in a perceptually heterogeneous mixture, and the locus of that mechanism, are as yet unknown. The present study was designed to explore the idea that mixture suppression is a perceptual phenomenon and not the result of physical, chemical, or receptor-substance interactions. An investigation was carried out as to whether perceptually similar taste stimuli give rise to the same sensory interactions when mixed with a substance of a different taste quality. In the first study, five different sweeteners (sucrose, fructose, aspartame, saccharin, and sorbitol) were matched in perceived sweetness intensity, in order to obtain five perceptually similar stimuli. Every equisweet sweetener concentration was mixed with each of four citric acid concentrations. In a second study, the sourness-suppressing effects of two sweeteners, sucrose and aspartame, were compared at four different concentration levels. Sourness scale values of unmixed citric acid, the unmixed sweeteners, and the citric acid/sweetener mixtures were assessed with a functional measurement approach in combination with a two. stimulus procedure. The equisweet sweeteners were equally effective in suppressing the perceived sourness intensity of citric acid over the concentration range used. The side tastes of the sweeteners, if present, did not have a substantial effect on the degree of sourness suppression.
\end{abstract}

When two or more substances of qualitatively different tastes are mixed, the overall perceived taste intensity of the mixture is, in most cases, less than the sum of the intensities of the unmixed components (see, e.g., Bartoshuk, 1975). This phenomenon, called mixture suppression, is the result of a decrease of the specific taste intensities (sweetness, sourness, saltiness, bitterness) contributing to the overall intensity of the solution (De Graaf \& Frijters, 1989; Schifferstein \& Frijters, 1990). The suppression of one taste quality by the other in a binary mixture is usually mutual, but not necessarily symmetrical. For example, Schifferstein and Frijters (1990) found that in a mixture of $1.00 \mathrm{M}$ sucrose and $0.010 \mathrm{M}$ citric acid, the sweetness intensity of sucrose was suppressed by 11 units, while the sourness intensity of citric acid was suppressed by 52 units. The total taste intensities of the unmixed stimuli were comparable in strength, being 73 and 79 units, respectively.

The exact mechanism that causes mixture suppression and the locus of that mechanism are as yet undetermined. Kroeze (1978) has shown that two phenomena that can

The authors gratefully acknowledge the funding provided by the Netherlands Organization for Scientific Research (NWO) for this project. The research was conducted while $H$. N. J. Schifferstein was supported by a NWO PSYCHON-grant No. 560-262-032, which had been awarded to J. E. R. Frijters. The authors also wish to thank Marjon Theunissen, Hilda Smit, and Liesbeth Hijwegen-Weemering for their assistance in collecting the data. Correspondence should be addressed to Hendrik N. J. Schifferstein, Department of Food Science, Agricultural University, Bomenweg 2, 6703 HD Wageningen, The Netherlands. cause a decrease in taste intensity, mixture suppression, and self-adaptation are independent and have to be accounted for by different processes. He demonstrated that there is no relation between a subject's saltiness score when the subject is given a sucrose $/ \mathrm{NaCl}$ mixture and the saltiness score when the subject is given a solution of unmixed $\mathrm{NaCl}$ after adaptation to $\mathrm{NaCl}$. In addition, he demonstrated that adaptation to unmixed $\mathrm{NaCl}$ and adaptation to a sucrose $/ \mathrm{NaCl}$ mixture, in which the saltiness intensity is suppressed by sucrose, both decreased the saltiness of $\mathrm{NaCl}$ to the same degree. Similar results were obtained for the sweetness of the $\mathrm{NaCl} /$ sucrose mixture (Kroeze, 1979). Kroeze concluded that self-adaptation and mixture suppression have different locations in the taste system and that mixture suppression is more centrally located than adaptation.

Since there are no structural elements of the neural part of the taste system that connect the two sides of the tongue before the thalamic level (Norgren \& Leonard, 1973), Kroeze and Bartoshuk (1985) carried out a split-tongue experiment to gain information about the locus of mixture suppression. They compared two conditions. In the first one, a mixture was applied to one tongue half while the other tongue half was stimulated with deionized water. In the second condition, the two components were spatially separated by applying each substance to a different tongue half. In quinineHCl/sucrose mixtures, they demonstrated that mixing quinine $\mathrm{HCl}$ with sucrose on the same tongue half, or administering the unmixed substances to the two tongue halves separately, decreased the bitterness to the same degree. This result suggests that quinine $\mathrm{HCl}-$ 
bitterness suppression by sucrose resides in thalamic or higher neural structures. In contrast, however, there was a 23\% decrease in bitterness intensity in the quinine$\mathrm{HCl} / \mathrm{NaCl}$ mixture when quinine $\mathrm{HCl}$ and $\mathrm{NaCl}$ were applied separately to the two tongue halves, compared to $69 \%$ when both substances were applied to the same tongue half. Therefore, Kroeze and Bartoshuk concluded that the observed decrease in bitterness intensity in quinine $\mathrm{HCl} / \mathrm{NaCl}$ mixtures results from both central and peripheral suppression mechanisms.

Gillan (1982) carried out an experiment in which he varied the distance between two areas of the human tongue stimulated by two substances of different taste qualities. $\mathrm{He}$ found that if the distance between the stimulated areas was enlarged, the decrease in taste intensity was diminished. This result shows that peripheral mechanisms are important in determining the degree of mixture suppression.

Several authors observed that after elimination or reduction of the taste intensity of one component, the taste sensation of the other component of a heterogeneous mixture is, to some degree, released from suppression. In some studies, this phenomenon was demonstrated with the use of a self- or cross-adaptation paradigm (e.g., Gillan, 1982; Kuznicki \& McCutcheon, 1979; Lawless, 1979). In other studies, the sweetness perception was blocked with Gymnema sylvestre (e.g., Lawless, 1979).

Kuznicki and McCutcheon (1979) observed suppression release in a sucrose $/ \mathrm{HCl}$ mixture after adaptation to sucrose following a pretreatment with Gymnema sylvestre. They concluded that mixture suppression and suppression release can apparently occur in $\mathrm{HCl}$ /sucrose mixtures without the involvement of the sweet taste system. It is possible, however, that the sweetness perception was not completely blocked in their experiment.

Kroeze (1982) used a habituation paradigm to study suppression release. He habituated subjects to the sweetness of sucrose or glucose and subsequently presented an $\mathrm{NaCl}$ /sucrose mixture. He demonstrated that the saltiness of the $\mathrm{NaCl}$ /sucrose mixture approximated the saltiness of the unmixed $\mathrm{NaCl}$ after repeated stimulation with sweet tasting stimuli. In a similar way, he demonstrated that the sweetness of sucrose in the sucrose $/ \mathrm{NaCl}$ mixture was released from suppression after repeated stimulations with unmixed $\mathrm{NaCl}$ (Kroeze, 1983). Since habituation is a central event, the mixture suppression found is also taken to be of a central origin.

In the present instance, mixture suppression is studied in a way different from the approach in previous studies. Here, we shall try to demonstrate that mixture suppression is a perceptual phenomenon and not the result of physical, chemical, or receptor-substance interactions. The following reasoning forms the basis of our thinking: Suppose there are two substances, $A$ and $B$, that elicit the same taste quality. If the taste intensity of a specified concentration of $B$ is matched to that of a certain concentration of $A$, one has obtained two stimuli that give rise to the same taste sensation, irrespective of their chemical structures. Assume further that there is another taste substance, $X$, which tastes qualitatively different from $A$ and $B$. If a certain concentration of $X$ is mixed with each of the equi-intense stimuli $A$ and $B$, one obtains two binary mixtures that are composed of perceptually similar components. If substance $X$ interacts in exactly the same way, or does not interact at all with $A$ and $B$ at the physical, chemical, or receptor level, then both the $X-A$ and the $X-B$ mixture should give rise to the same taste sensations and the same sensory interactions. If $A$ and $B$ are two substances that differ with regard to chemical structure and concentration level, it does not seem likely that both substances will exhibit the same interactions in the mixture or at the receptor site. Therefore, it seems plausible to assume that if two chemically different, but perceptually similar stimuli give rise to the same sensory interactions, the locus of the interaction mechanism must reside in the afferent pathways, the central neural system, or at the level of conscious experience-that is, the percept.

In 1965, Pangborn reported the results of a study in which equi-intense stimulus concentrations were used to study mixture suppression. Specifically, she investigated the effect of four organic acids on the sweetness intensity of four sugars. The sugar concentrations were equivalent in sweetness intensity, and the acid concentrations were equivalent in sourness intensity. Pangborn reported that citric acid and acetic acid usually exhibited greater sweetness-suppressing action than did lactic and tartaric acids when they were mixed with one of the four sugars in a binary mixture. However, since it was not her objective to demonstrate that equisour acids produce the same degree of sweetness suppression in a sugar/acid mixture, she did not test whether the differences between these four acids were significant.

In the first study, the influence of five different sweeteners on the degree of sourness suppression in citric acid/sweetener mixtures was assessed. Citric acid/ sweetener mixtures were chosen, because the sourness of a citric acid/sucrose mixture is highly dependent on both the citric acid and the sucrose concentration (Schifferstein \& Frijters, 1990). The five sweeteners were chosen as representative of a range of chemically different substances: aspartame (dipeptide), saccharin (imide), sorbitol (sugaralcohol), fructose (monosaccharide), and sucrose (disaccharide). The five concentrations of sweeteners were preexperimentally equated, with regard to sweetness intensity, to obtain five perceptually similar stimuli. Subsequently, the obtained sweetener concentrations were used to construct five stimulus series. In each series of citric acid/sweetener mixtures, the citric acid concentration was the only variable, while the sweetener concentration remained constant.

A second study was carried out to investigate whether two sweeteners (aspartame and sucrose) suppressed the sourness of citric acid to the same degree at four different sweetener levels. Four sucrose concentrations were matched in perceived sweetness intensity to four aspartame levels, and the obtained concentrations were used 
to construct eight series of citric acid/sweetener mixtures. In every series, the citric acid concentration varied while the sweetener concentration remained constant.

For one to draw proper conclusions about the degrees to which the different sweeteners suppress sourness, one must assess the perceived sourness intensities on an interval or ratio scale. Whether or not the derived scale has interval properties depends on the validity of the assumption that the scale values derived from the overt responses vary linearly with the perceived taste intensities. This assumption can be verified with a functional measurement approach in combination with a two-stimulus procedure (De Graaf, Frijters, \& van Trijp, 1987).

One of the main features of functional measurement is the use of factorial designs as a tool for the evaluation of the judgment function. Two different factorial designs can be distinguished: a factorial judgment design and a factorial mixing design. In a factorial judgment design, the subjects compare the sensory impression of each level of a column stimulus with the impression of each level of a row stimulus. Parallelism in the factorial plot of the obtained data does not depend on sensory interaction but on the nature of the comparative operation and on the form of the judgment function. If subjects are instructed to judge differences, the comparative operation between two sensory impressions can be best described with an algebraic subtractive rule. The subtractive model predicts no interaction between row and column stimuli, assuming that the response scale is linear (e.g., Birnbaum, 1978; Birnbaum \& Mellers, 1978). Therefore, testing the row $x$ column interaction for significance provides the basis for testing the linearity of the response scale. If the response scale is linear, the marginal means of the row and column stimuli are validated estimates of the perceived taste intensity of the corresponding row and column stimuli on an interval scale (De Graaf et al., 1987).

In a factorial mixing design (De Graaf et al., 1987; McBride, 1986), each level of the first factor (type of sweetener) is mixed with each level of the second factor (citric acid concentration). The stimuli resulting from the factorial mixing design can be incorporated in the factorial judgment designs. If the judgment function is linear, parallelism in a factorial plot of mixture components indicates that the components behave additively.

The term factorial mixing design refers to the physical composition of mixtures and should not be confused with the term factorial judgment design, which refers to the presentation of pairs of (mixed and unmixed) stimuli to the subjects (De Graaf et al., 1987).

In the present studies, it is assumed that tasting a citric acid/sweetener mixture leads to the formation of a heterogeneous percept. Subjects can reliably analyze this percept in order to give estimates of the perceived sourness intensity and the perceived sweetness intensity. Evidence for the validity of this assumption has been provided by Schifferstein and Frijters (1990), who demonstrated that the weighted sum of sweetness and sourness equals the total taste intensity of a citric acid/sucrose mixture.

\section{EXPERIMENT 1}

\section{Method}

Subjects. The subjects were 14 paid volunteers, 8 women and 6 men, ranging in age from 19 to 27 years. They were under-graduate students at the Agricultural University. All subjects had had previous experience with psychophysical tasks, but all were naive with respect to the substances used and the purpose of the study.

Stimuli. The stimuli were solutions of citric acid (Merck 244), sucrose (Merck 7651), aspartame (Holland Sweetener Company), saccharin (Sigma S-1002), fructose (Merck 5321), and sorbitol (BDH 30242), as well as mixtures of these sweeteners with citric acid in demineralized water. The concentrations of citric acid were $0.00,0.00125,0.0025,0.005$, and $0.010 \mathrm{M}$ citric acid.

In a preliminary experiment, aspartame, saccharin, fructose, and sorbitol were matched in perceived sweetness intensity to $0.25 \mathrm{M}$ sucrose, using the method of constant stimuli (De Graaf \& Frijters, 1986). Thirteen of the 14 subjects participated in this experiment. The concentrations that were determined as equisweet to $0.25 \mathrm{M}$ sucrose were $0.0030 \mathrm{M}$ aspartame (corrected for the water content), $0.0020 \mathrm{M}$ saccharin, $0.3891 \mathrm{M}$ fructose, and $0.8346 \mathrm{M}$ sorbitol.

The mixtures were constructed on the basis of a factorial mixing design. Every sweetener was mixed with each of the citric acid concentrations.

For reasons of standardization, the subjects were presented with a reference pair. The first stimulus of the reference pair was water; the second was $0.0125 \mathrm{M}$ citric acid.

All solutions were prepared at least $24 \mathrm{~h}$ prior to tasting and were stored at $4^{\circ} \mathrm{C}$ for a period not longer than 1 week.

Design. The investigation consisted of 12 experiments, each of which employed a factorial judgment design. A factorial judgment design implies that subjects are presented with pairs of stimuli. In each investigation, an $m \times n$ design is employed, where $m$ and $n$ denote the number of concentrations of the first (row) and second (column) stimulus. In order to incorporate all the experimental stimuli in factorial judgment designs, six series of stimuli were constructed. One series was constructed for each of the five sweeteners. Each series consisted of four citric acid/sweetener mixtures, a solution of unmixed sweetener, and water (six stimuli). The sixth series consisted of the four unmixed citric acid concentrations $(0.00125,0.0025,0.005$, and $0.010 \mathrm{M})$ and water (five stimuli). Each of the six series of experimental stimuli was compared with regard to sourness intensity with three citric acid solutions: 0.00 (water), 0.0025 , and $0.010 \mathrm{M}$ citric acid. In six experiments, the three comparison stimuli were presented as first stimuli; in six other experiments they served as second stimuli.

Procedure. The subjects were instructed to judge the magnitude of the difference in perceived sourness intensities between the first and second stimulus of each pair. The instructions emphasized that only the sourness intensity should be judged.

The judgments were expressed by a slash mark on a $250-\mathrm{mm}$ visual analogue scale. The middle of the scale was defined thus: "The first and second stimulus are equal with respect to perceived sourness intensity" (see De Graaf et al., 1987; Figure 3). If the first stimulus was perceived as tasting more sour than the second stimulus, the subject placed a mark on the left side of the scale. The subject marked the right side of the scale when the second stimulus was perceived as being more sour. The distance between the slash mark and the middle of the scale indicated the size of the intensity difference beween the two samples of one pair. The left end 
and the right end of the scale were labeled "maximum difference." In the instructions, "maximum difference" was defined as the difference in sourness intensity between the stimuli of the reference pair (i.e., water as the first stimulus and $0.0125 \mathrm{M}$ citric acid as the second stimulus). The difference between the stimuli of the reference pair was expected to be larger than the difference in any other pair. A response was measured as the distance in millimeters from the left pole of the scale. A response value of 125 meant no difference, a value above 125 meant that the second stimulus was perceived as being more sour than the first, and a value below 125 indicated that the first stimulus was perceived as being the most sour of the pair.

The subjects were requested to rinse their mouths thoroughly with demineralized water, both within and between pairs. The stimuli were presented at room temperature in polystyrene medicine cups that contained about $10 \mathrm{ml}$ of solution. The pairs of each factorial design were presented in a random sequence and in a different order for each subject. The reference pair was presented at the beginning of each session, and again after the 12th and 24th pair of each session. The time interval between and within pairs was $40 \mathrm{sec}$. Each of the 12 factorial judgment designs was presented to each subject twice. It took each subject 1250 -min sessions to complete the entire investigation.

\section{Results}

A procedure recently developed by De Graaf et al. (1987) and De Graaf and Frijters (1988) was applied to the present data. A brief outline of the psychometric properties of the response scale and an explanation of how scale values were derived is given in the next section.

Psychometric properties of response scale and derivation of scale values. The subjects were instructed to judge the difference between the perceived taste intensities within each pair of stimuli. Parallelism in a factorial judgment plot implies that the response function is linear with the perceived difference. Because individuals may vary in their judgment functions (linear or nonlinear), or in their comparative operations (which may or may not be subtractive), individual analyses were performed first. The measure of the degree of nonparallelism - that is, the row $\times$ column interaction-was tested for statistical significance in an analysis of variance, with the row $x$ column $\times$ replication interaction as an error term. Out of 168 analyses, none showed a significant row $\times$ column (first stimulus $\times$ second stimulus) interaction $(p>.01)$.

At group level, the row $x$ column interaction was tested for significance against the row $\times$ column $\times$ subject interaction for each of the 12 factorial judgment designs. The interaction appeared to be significant in one case only $(p<.01)$.

The number of significant interactions approximates the number that may be expected to reach significance given this number of analyses and the specified alpha level. Therefore, it can be concluded that the responses obtained are a linear function of perceived taste intensity differences. Consequently, the marginal means of the row and column stimuli are validated estimates of the perceived taste intensity on an interval scale (Anderson, 1981).

If it is assumed that water has no taste, then scale values can be derived for each of the experimental stimuli by calculating the difference between the marginal mean for the experimental stimulus and the marginal mean for water in each of the 12 experiments. The final scale value for each stimulus was calculated by averaging the scale value for that stimulus tasted as first stimulus of each pair and the scale value when tasted as second stimulus of each pair. All data were averaged over subjects and replications.

Comparison of the sweeteners with regard to sourness suppression. Figure 1 shows the sourness of citric acid and the citric acid/sweetener mixtures as a function of the sourness of citric acid with a separate curve for each mixture type. Visual inspection shows that the sourness intensity of all citric acid/sweetener mixtures is lower than the sourness of the corresponding unmixed citric acid concentrations in all cases.

If an acid is mixed with a number of different concentrations of the same sweetener, a plot of diverging curves is observed (e.g., see Figure 4). The sourness of each $\mathrm{acid} /$ sweetener mixture depends on both the acid and the sweetener concentration. If the sourness of citric acid is differentially suppressed by each of the five equisweet sweeteners used in this study, a similar diverging pattern is to be expected. However, if the five sweeteners are equally effective in suppressing the sourness of citric acid, the five mixture curves should coincide. Figure 1 shows that the differences in the degrees of sourness suppression caused by the five equisweet sweeteners were not comparable to those caused by different concentrations of the same sweetener. In contrast, the five mixture functions are similarly shaped and differ only slightly. Analysis of variance of the five citric acid/sweetener mixtures shows significant effects for the citric acid concentration $[F(4,52)=109.68, p<.001]$ and the sweetener type $[F(4,52)=2.66, p=.04]$. The sweetener $\times$ citric acid interaction is not significant $[F(16,208)=0.69, p=.80]$.

The significance of the sweetener type effect is largely due to the aspartame/citric acid mixtures that have consistently higher scale values than the other mixture types. If the aspartame data are excluded from the analysis, the observed sweetener effect is no longer significant $[F(3,39)=0.88, p=.46]$.

The higher sourness scale values for the aspartame mixtures may result from the sourness of the unmixed sweetener. The sourness scale value of $0.0030 \mathrm{M}$ unmixed aspartame differs almost significantly from zero [onetailed $t$ test, $p=.07$ ]. Therefore, the aspartame curve may lie higher than the other curves, because the sourness intensity of the unmixed aspartame may have been added to the sourness of citric acid at each citric acid level. This could be a plausible explanation, since the significance of the sweetener effect disappears if the sourness scale value of each unmixed sweetener is subtracted from the scale value of each mixture of that sweetener with citric acid $[F(4,52)=1.25, p=.30]$.

It may seem as if the differences between sweeteners are consistent over concentrations because the aspartame mixtures have higher scale values (at four citric acid levels) and the sorbitol mixtures have lower scale values (at three citric acid levels) than the other mixtures. Such consistent differences can be the consequence of the calculation method employed. The scale value of each stimu- 


\section{sourness citric acid/sweetener mixture}

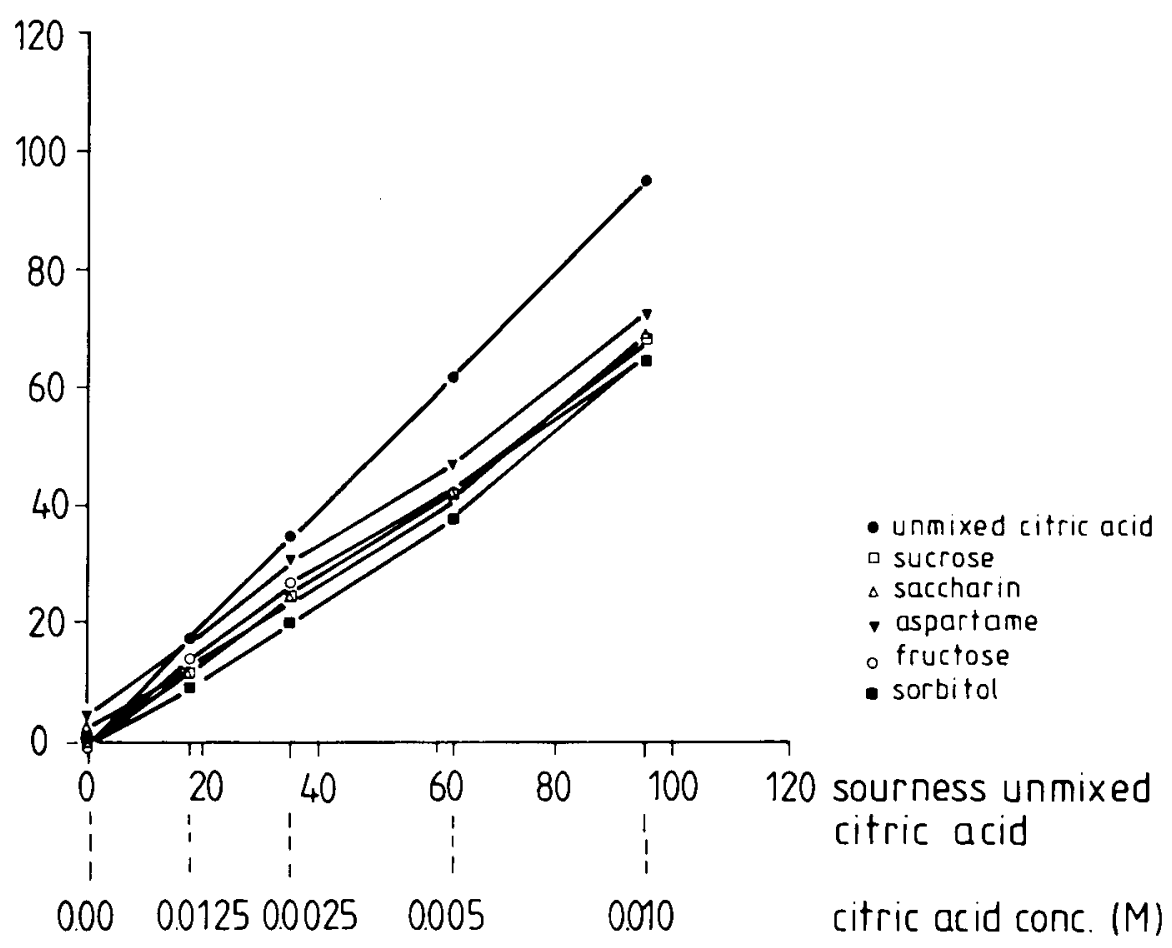

Figure 1. The perceived sourness intensity of citric acid/sweetener mixtures, plotted as a function of the sourness of unmixed citric acid with a separate curve for every sweetener.

lus is derived by calculating the difference between the marginal mean for that stimulus and the marginal mean for water, which is used as a rational zero point. Therefore, the marginal mean for water determines the position of the scale values on the ordinate. For each of the six factorial judgment designs, one marginal mean is calculated for water. This marginal mean is not the same for each sweetener, but rather is subject to some random variation (standard error of mean $\approx 4$ ). Because the scale values for each sweetener are calculated from a different factorial judgment design, a set of slightly differing but parallel curves may appear instead of a set of coinciding curves.

In summary, it can be concluded that four of the five mixture functions in Figure 1 do not differ statistically. The deviance of the aspartame curve might be due to a sour side-taste of the unmixed aspartame at the concentration level used in this experiment and/or to an artifact of the calculation method employed.

\section{EXPERIMENT 2}

\section{Method}

Subjects. The subjects were 14 paid volunteers, 11 women and 3 men, whose ages ranged from 20 to 26 years. All subjects were undergraduate students at the Agricultural University. All subjects had had previous experience with psychophysical tasks, but were naive with respect to the substances used and the purpose of the study.
Stimuli. The stimuli were solutions of aspartame (Holland Sweetener Company), sucrose (Merck 7651), citric acid (Merck 244), and citric acid/sweetener mixtures in demineralized water.

In a preliminary experiment, sucrose was matched as regards perceived sweetness intensity with $0.0003,0.0010,0.0032$, and $0.0102 \mathrm{M}$ aspartame, using the method of constant stimuli (De Graaf \& Frijters, 1986). In this experiment, all 14 subjects participated. The concentrations that were determined as being equisweet to the aspartame concentrations were $0.05,0.14,0.27$, and $0.44 \mathrm{M}$ sucrose. The citric acid concentrations were $0.00,0.00125,0.0025$, 0.005 , and $0.010 \mathrm{M}$ citric acid.

Every sweetener concentration was mixed with every citric acid level in order to obtain eight different series. Within every series, the citric acid level varied while the sweetener concentration remained constant. Every mixture series consisted of four citric acid/sweetener mixtures, the unmixed sweetener, and water. In addition to the mixture series, a series of unmixed citric acid stimuli was used. The reference pair for the sourness investigation consisted of water and $0.0125 \mathrm{M}$ citric acid.

In a separate investigation, the sweetness of the unmixed sweetener concentrations was assessed. In this investigation, the reference pair consisted of water as first stimulus and $1.25 \mathrm{M}$ sucrose as second stimulus.

The solutions were prepared at least $24 \mathrm{~h}$ before tasting and were stored at $4^{\circ} \mathrm{C}$ for a period not longer than 1 week.

Design. The sourness investigation consisted of 18 experiments, each of which employed a factorial judgment design. There were $166 \times 3$ and $25 \times 3$ factorial judgment designs. In order to incorporate all the experimental stimuli in factorial judgment designs, nine series of stimuli were constructed: one series of unmixed citric acid solutions (five stimuli) and eight series of citric acid/sweetener mixtures (six stimuli). Water was included in each of these series 
as a meaningful zero point to be used in the calculations of scale values.

Each of the nine series of experimental stimuli was compared with regard to sourness intensity with three citric acid solutions: 0.00 (water), 0.0025 , and $0.0100 \mathrm{M}$ citric acid. These three solutions were presented as first stimuli in nine experiments and as second stimuli in nine other experiments.

To check the perceptual similarity of the unmixed aspartame and sucrose levels, the perceived sweetness intensities of the unmixed sweetener levels were determined in a separate investigation. This sweetness investigation consisted of four experiments, each of which employed a $5 \times 3$ factorial judgment design. There were two series of five experimental stimuli: one series of aspartame solutions and one series of sucrose solutions. Water was included in both series as a meaningful zero point.

The two series were compared with regard to perceived sweetness intensity with three sucrose solutions: 0.00 (water), 0.25 , and $1.00 \mathrm{M}$ sucrose. Each of these solutions was presented as the first stimulus in two experiments and as the second stimulus in the two other experiments.

Procedure. The procedure was identical to the procedure used in the first study. In the sweetness investigation, however, the magnitude of the difference in perceived sweetness intensities had to be judged.

The 22 experiments ( 18 sourness and 4 sweetness experiments) were presented to each subject twice. During each session, all pairs of two designs were judged. The order of presentation of the designs was random, with the restriction that two designs, which were to be presented simultaneously, had to be of the same type (sourness or sweetness experiment). It took each subject 22 50-min sessions to complete the entire investigation.

\section{Results}

Psychometric properties of the response scale and derivation of the scale values. In order to check the linearity of the response function, analyses of variance were carried out for each individual subject for each factorial judgment design $(14 \times 22=308$ analyses $)$. The row $x$ column interaction was found to be significant in only four cases $(p<.01)$. None of the subjects had more than one significant interaction $(p<.01)$. On group level (22 analyses), the row $\times$ column interaction was not significant in any of the cases $(p>.01)$.

Since the number of significant interactions approaches the number that may be expected to reach significance given this number of analyses, it can be concluded that the responses obtained are a linear function of the perceived taste intensity differences. Consequently, the marginal means of the row and column stimuli are validated estimates of the perceived taste intensity on an interval scale (Anderson, 1981). The procedure that was followed to calculate the scale values was identical to the one described in the first study.

Sweetness of the unmixed sweeteners. The psychophysical functions for aspartame and sucrose have been reproduced in Figure 2 . The aspartame function accelerates negatively over the whole concentration range, while the sucrose function accelerates positively at low concentration levels and accelerates negatively at high concentration levels. The correspondence between the sweetness intensities at the four different concentration levels is almost perfect $(r=1.00)$. The concentrations of aspartame and sucrose obtained in the matching experiment appear to be equisweet (Figure 3). Analysis of variance of the sweetness data showed that sweetener type had no significant effect $[F(1,13)=0.11, p=.75]$.

Comparison of the sweeteners with regard to sourness suppression. Figure 4 (panels $A$ and $B$ ) shows the sourness of citric acid and the sweetener/citric acid mixtures as a function of the sourness of citric acid, with a separate curve for each sweetener concentration. Visual inspection shows two highly similar plots of diverging functions, in which higher sweetener concentrations obviously produce a greater sourness-suppressing effect. In addition, the citric acid concentration influences the sourness intensity and the degree to which it is suppressed.

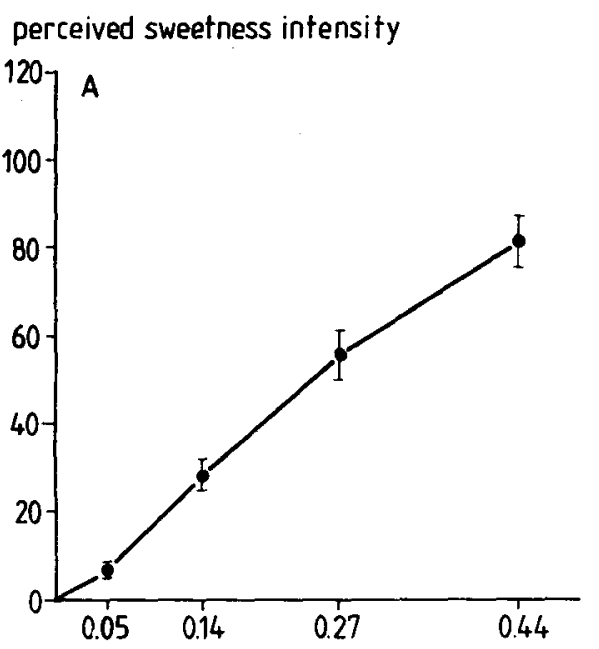

sucrose concentration (M)

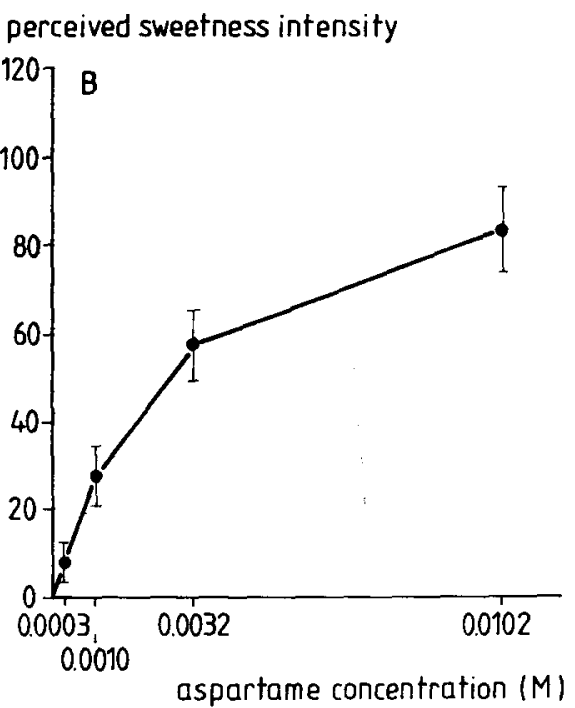

Figure 2. Psychophysical functions for the sweetness intensities of sucrose (panel A) and aspartame (panel B). The error bars around each point represent the $95 \%$ confidence interval for each scale value. 


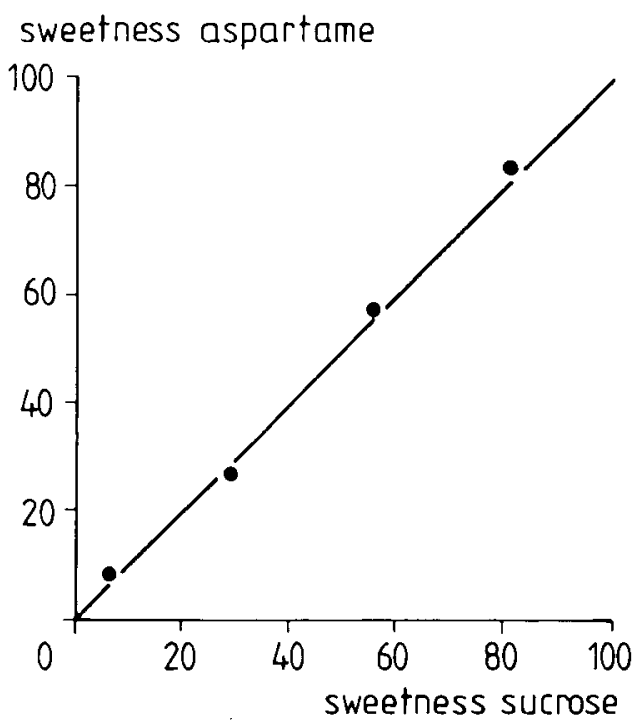

.Figure 3. The relationship between the sweetness of aspartame and the sweetness of sucrose. Points are located on the diagonal if the aspartame and sucrose concentrations are equisweet.
Analysis of variance of the data of the aspartame mixtures showed significant effects for the citric acid concentration $[F(4,52)=889.61, p<.001]$, the aspartame concentration $[F(3,39)=118.71, p<.001]$, and the aspartame $\times$ citric acid interaction $[F(12,156)=19.03$, $p<.001]$. Analysis of the data for the sucrose mixtures gave similar results. The citric acid concentration $[F(4,52)$ $=1,227.52, p<.001]$ and the sucrose concentration $[F(3,39)=85.17, p<.001]$ showed significant effects, and the sucrose $\times$ citric acid interaction $[F(12,156)=$ $9.15, p<.001]$ was also significant. These results indicate that the degree of soumess suppression depends on both the citric acid concentration and the sweetener level.

The type of sweetener has no effect on the sourness intensity or on the degree of sourness suppression, however. An analysis of variance of the entire data set reveals neither a significant effect of the type of sweetener $[F(1,13)=0.00, p=.98]$ nor a significant sweetener type $\times$ citric acid interaction $[F(4,52)=1.83, p=.14]$. Figure 5 shows the relationship between the sourness of the aspartame/citric acid mixtures and the sourness of the sucrose/citric acid mixtures. These results clearly indicate that both sweeteners suppress the sourness of citric acid to the same degree $(r=.98)$.

\section{sourness sucrose / citric acid mixture}

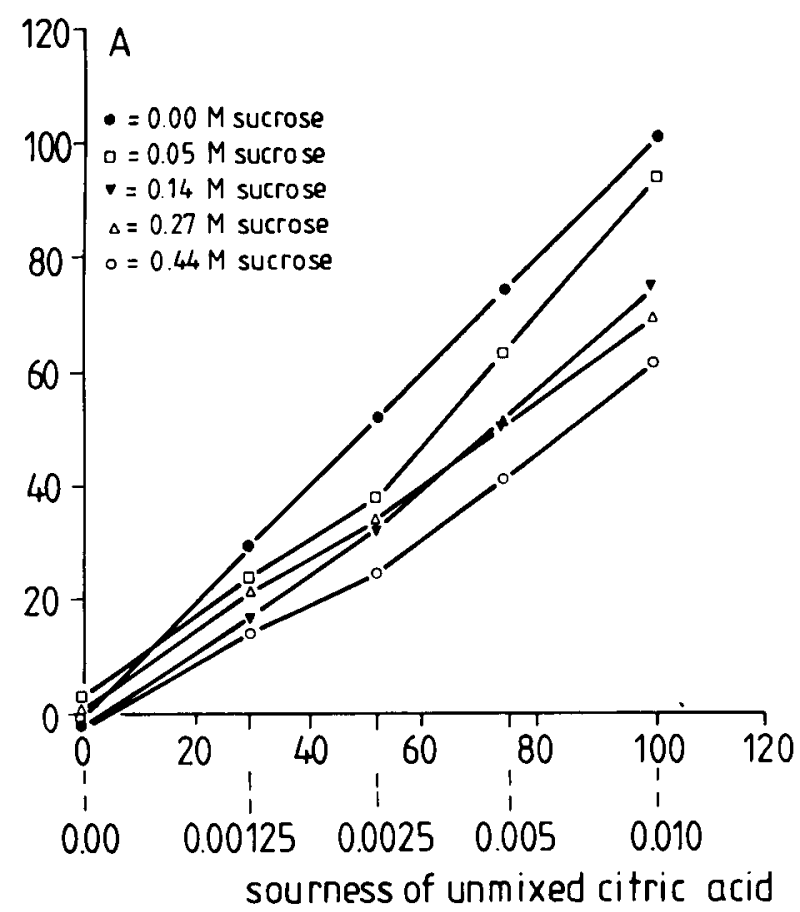

\section{sourness aspartame/citric acid mixture}

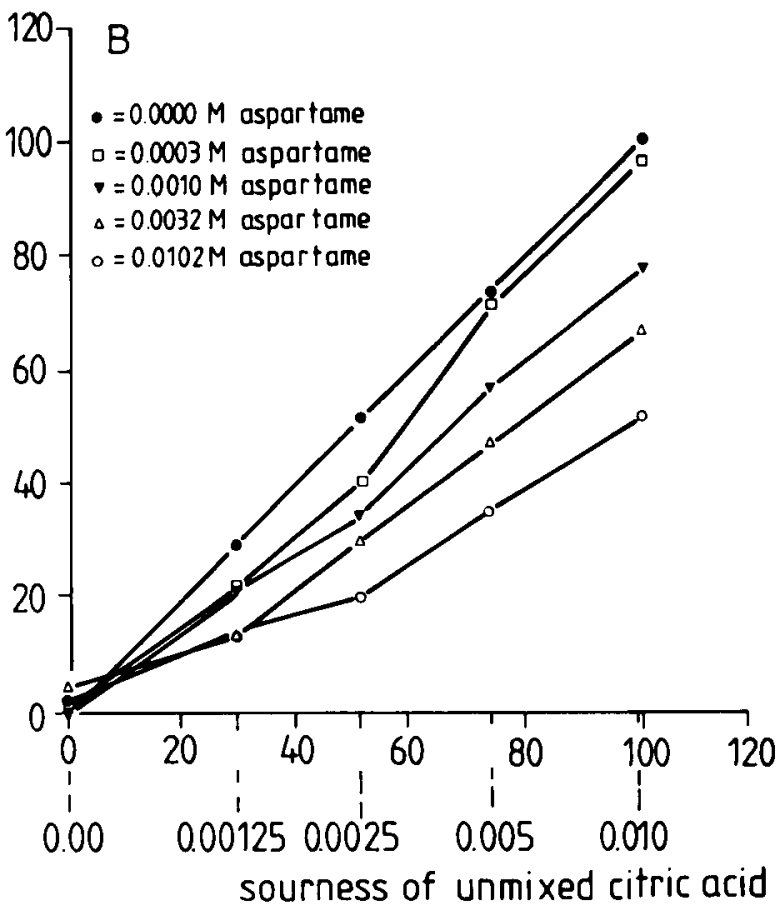

Figure 4. The sourness intensity of citric acid and the citric acid/sweetener mixtures as a function of the sourness of unmixed citric acid, with a separate curve for each sweetener concentration. Panel A shows the sourness of the sucrose/citric acid mixtures. Panel B shows the sourness of the aspartame/citric acid mixtures. 


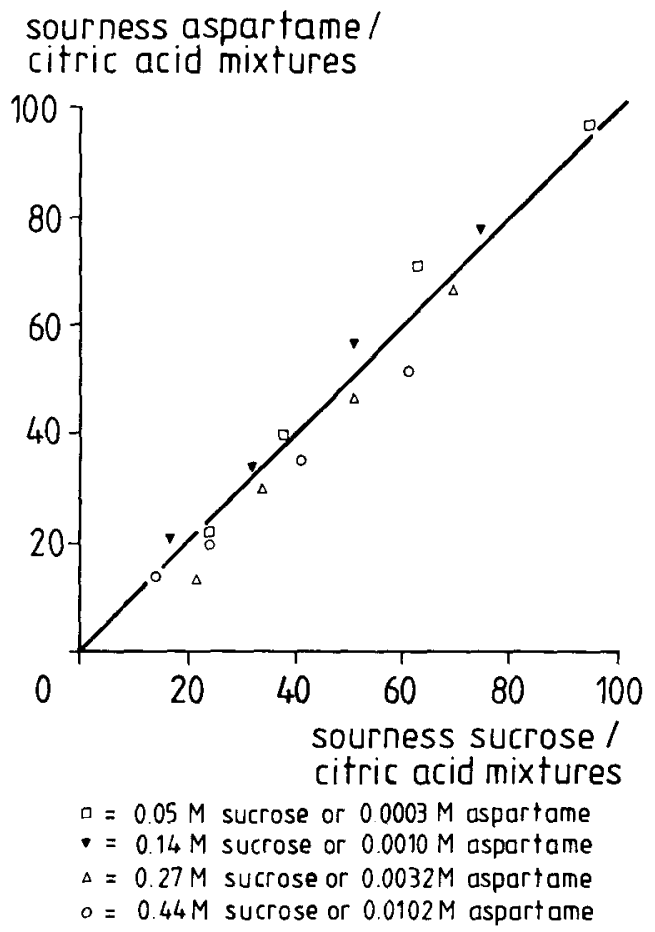

Figure 5. The relationship between the sourness of the citric acid/sucrose mixtures and the sourness of the citric acid/aspartame mixtures. Points are located on the diagonal if the sucrose mixtures and the aspartame mixtures are equisour. The citric acid concentrations were $0.00125,0.0025,0.005$, and $0.010 \mathrm{M}$ in all cases.

It should be noted that unmixed aspartame has a slight positive sourness value at each of the four concentration levels. The sourness value of $0.0032 \mathrm{M}$ aspartame is significantly different from zero $(p<.05)$. Unlike the results of the first study, however, the scale values of the $0.0032 \mathrm{M}$ aspartame/citric acid mixtures are not consistently higher than those for the mixtures containing $0.27 \mathrm{M}$ sucrose.

\section{DISCUSSION}

\section{Side Tastes}

Aspartame was found to elicit a slight sour side taste at $0.0030 \mathrm{M}$ (first study) and $0.0032 \mathrm{M}$ (second study). Schiffman, Reilly, and Clark (1979) reported that several subjects detected a bitter component in the aspartame taste, which developed with time. Some of the graphs and tables published by Larson-Powers and Pangborn (1978b) show that aspartame tastes slightly more bitter and sour than sucrose. Nevertheless, these authors agree with most of their colleagues that aspartame tastes much like sucrose (e.g., Larson-Powers \& Pangborn, 1978a, 1978b; Schiffman, Crofton, \& Beeker, 1985).

Saccharin is known to elicit a bitter side taste, especially at high concentration levels (e.g., Larson-Powers \& Pangborn, 1978a, 1978b; Moskowitz, 1970; Moskowitz \& Klarman, 1975; Schiffman et al., 1985; Schiff- man et al., 1979), and sometimes it has been reported to have a sour side taste (e.g., Larson-Powers \& Pangborn, 1978a, 1978b; Schiffman et al., 1985).

In the present instance, the sourness intensity of $0.0030 \mathrm{M}$ aspartame seems to have influenced the results of the first study, because the scale values of the aspartame/citric acid mixtures were consistently higher than the scale values of the other mixtures. In the second study, however, citric acid/aspartame mixtures and citric acid/ sucrose mixtures elicited the same sourness intensity. Saccharin, which is known to elicit more intense side tastes than aspartame (e.g., Larson-Powers \& Pangborn, 1978b), did not deviate substantially from the other sweeteners in the first study. It seems therefore, reasonable to conclude that side tastes do not affect the degree to which sweeteners suppress the sourness intensity of citric acid.

Mixture suppression: Central or peripheral? According to Kroeze (1989), there is no simple answer to the question of whether mixture suppression is a peripheral or central phenomenon, since the afferent sensory system should be regarded as a continuous pathway between receptor sites and the locus of sensation. Furthermore, perception should be regarded as an active process in which feedback channels may play an important role by inducing peripheral sensory changes and causing subjects to display selective attention.

The objective in the present study was to investigate whether perceptually equal stimuli give rise to the same sensory interactions. The results demonstrated that equisweet solutions produce the same degree of sourness suppression when mixed with the same quantity of acid for the concentration ranges used in the two experiments. This outcome makes it very unlikely that mixture suppression can be accounted for by chemical or receptor events. This conclusion can be substantiated by the following example. Sucrose and aspartame are two, chemically entirely different, substances. They elicit the same sweetness intensities at completely different concentration levels. For instance, according to the results of the second study, $0.14 \mathrm{M}$ sucrose is equisweet to $0.0010 \mathrm{M}$ aspartame. If one tries to account for the sourness suppression in a citric acid/sweetener mixture by referring to the protonaccepting capacity of the sucrose molecule (Kuznicki \& McCutcheon, 1979), it follows from the present results that one molecule of aspartame should accept about 140 times the number of protons a sucrose molecule attracts, which is very unlikely.

Sweet substances and the ways in which these substances are perceived can be very different. In the present study, several sweet substances were used, with different chemical structures, different concentration levels, and unequally shaped psychophysical functions for the ranges of concentration (Figure 2). Several authors have suggested that sweet substances might stimulate different types of receptor sites to some degree (e.g., De Graaf \& Frijters, 1986; Lawless \& Stevens, 1983; McBride, 1988 ). Perhaps two sweetness messages may be processed 
in a different manner in the peripheral neural system. The present study has demonstrated that, in spite of all these differences, two sweeteners that taste equally sweet will always give rise to the same degree of sourness suppression. Therefore, it seems plausible to conclude that, since perceptually equal stimuli give rise to the same degree of mixture suppression when mixed with the same amount of acid, sourness suppression in citric acid/sweetener mixtures must take place at the perceptual level and not in the solution or at the receptor.

In a recently published study, McBride and Finlay (1990) reported that sucrose suppresses the sourness intensity of citric acid more effectively than fructose does. It was not mentioned whether this difference was statistically significant. In our study, however, equisweet concentrations of fructose and sucrose were shown to be equally effective in suppressing the sourness intensity of citric acid, since their curves in Figure 1 coincide. It should be noted that McBride and Finlay (1990) used a much higher citric acid concentration in their experiment $(0.050 \mathrm{M})$ than the highest concentration that was used in the present experiment $(0.01 \mathrm{M})$. Such an increase in citric acid level and the corresponding increase in sourness intensity not only could affect the perceptual process but also might lead to chemical changes in the fructose or sucrose solutions because of the low pH value (Shallenberger \& Birch, 1975).

\section{REFERENCES}

ANDERSON, N. H. (1981). Foundations of information integration the ory. New York: Academic Press.

Bartoshuk, L. M. (1975). Taste mixtures: Is mixture suppression related to compression? Physiology \& Behavior, 14, 643-649.

BirnBAUm, M. H. (1978). Differences and ratios in psychological measurement. In N. J. Castellan, Jr., \& F. Restle (Eds.), Cognitive the ory (Vol. 3, pp. 33-74). Hillsdale, NJ: Erlbaum.

Birnbaum, M. H., Mellers, B. A. (1978). Measurement and the mental map. Perception \& Psychophysics, 23, 403-408.

De GraAf, C., \& Fruters, J. E. R. (1986). A psychophysical investigation of Beidler's mixture equation. Chemical Senses, 11, 295-314.

De GraAf, C., \& Friuters, J. E. R. (1988). Assessment of the taste interaction between two qualitatively similar taste substances: A comparison between comparison rules. Joumal of Experimental Psychology: Human Perception \& Performance, 14, 526-538.

De GraAF, C., FriJters, J. E. R. (1989). Intertelationships among sweetness, saltiness and total taste intensity of sucrose, $\mathrm{NaCl}$, and sucrose/ $\mathrm{NaCl}$ mixtures. Chemical Senses, 14, 81-102.

De Graaf, C., Frujters, J. E. R., \& van Trujp, H. C. M. (1987). Taste interaction between glucose and fructose assessed by functional measurement. Perception \& Psychophysics, 41, 383-392.

GiLlAN, D. J. (1982). Mixture suppression: The effect of spatial separation between sucrose and $\mathrm{NaCl}$. Perception \& Psychophysics, 32 , 504-510.

Kroeze, J. H. A. (1978). The taste of sodium chloride: Masking and adaptation. Chemical Senses \& Flavour, 3, 443-449.
Kroeze, J. H. A. (1979). Masking and adaptation of sugar sweetness intensity. Physiology \& Behavior, 22, 347-351.

Kroeze, J. H. A. (1982). After repetitious sucrose stimulation saltiness suppression in $\mathrm{NaCl}$-sucrose mixtures is diminished: Implications for a central mixture suppression mechanism. Chemical Senses, 7, 81-92

Kroeze, J. H. A. (1983). Successive contrast cannot explain suppression release after repetitious exposure to one of the components of a taste mixture. Chemical Senses, 8, 211-223.

KROEZE, J. H. A. (1989). Is taste mixture suppression a peripheral or central event? In D. G. Laing, W. S. Cain, R. L. McBride, \& B. W. Ache (Eds.), Perception of complex smells and tastes (pp. 225-243). Sydney: Academic Press.

Kroeze, J. H. A., BArtoshuk, L. M. (1985). Bitterness suppression as revealed by split-tongue taste stimulation in humans. Physiology \& Behavior, 35, 779-783.

KUZNICKI, J. T., MCCUTChEON, N. B. (1979). Cross-enhancement of the sour taste on single human taste papillae. Joumal of Experimental Psychology: General, 108, 68-89.

Larson-Powers, N., Pangborn, R.M. (1978a). Descriptive analysis of the sensory properties of beverages and gelatins containing sucrose or synthetic sweeteners. Journal of Food Science, 43. 47-51.

LARson-Powers, N., \& PANGborn, R. M. (1978b). Paired comparison and time-intensity measurements of the sensory properties of beverages and gelatins containing sucrose or synthetic sweeteners. Journal of Food Science, 43, 41-46.

LAWLESS, H. T. (1979). Evidence for neural inhibition in bittersweet taste mixtures. Journal of Comparative \& Physiological Psychology, 93, 538-547.

Lawless, H. T., Stevens, D. A. (1983). Cross adaptation of sucrose and intensive sweeteners. Chemical Senses, 7, 309-315.

MCBRIDE, R. L. (1986). The sweetness of binary mixtures of sucrose, fructose and glucose. Journal of Experimental Psychology: Human Perception \& Performance, 12, 584-591.

MCBRIDE, R. L. (1988). Taste reception of binary sugar mixtures: Psychophysical comparison of two models. Perception \& Psychophysics, 44, 167-171.

MCBride, R. L., Finlay, D. C. (1990). Perceptual integration of tertiary taste mixtures. Perception \& Psychophysics, 48, 326-330.

Moskowitz, H. R. (1970). Sweetness and intensity of artificial sweeteners. Perception \& Psychophysics, 8, 40-42.

Moskowitz, H. R., Klarman, L. (1975). The tastes of artificial sweeteners and their mixtures. Chemical Senses \& Flavour, 1 , $411-421$

Norgren, R., \& LeonARD, C. M. (1973). Ascending central gustatory pathways. Joumal of Comparative Neurology, 150, 217-238.

PANGBORN, R. M. (1965). Taste intertelationships of organic acids and selected sugars. In J. M. Leitch (Ed.), Food science and technology: Vol. 3. Quality, analysis and composition of foods (pp. 291-305). London: Gordon \& Breach.

Schifferstein, H. N. J., \& Fruters, J. E. R. (1990). Sensory integration in citric acid/sucrose mixtures. Chemical Senses, 15, 87-109.

SChiffman, S. S., Crofton, V. A., Beeker, T. G. (1985). Sensory evaluation of soft drinks with various sweeteners. Physiology \& Behavior, 34, 369-377.

Schiffman, S. S., Reilly, D. A., Clark, T. B., III. (1979). Qualitative differences among sweeteners. Physiology \& Behavior, 23, 1-9.

ShAllenberger, R. S., \& BiRCh, G. G. (1975). Sugar chemistry. Westport, CT: AVI Publishing.

(Manuscript received March 19, 1990; revision accepted for publication August 6, 1990.) 